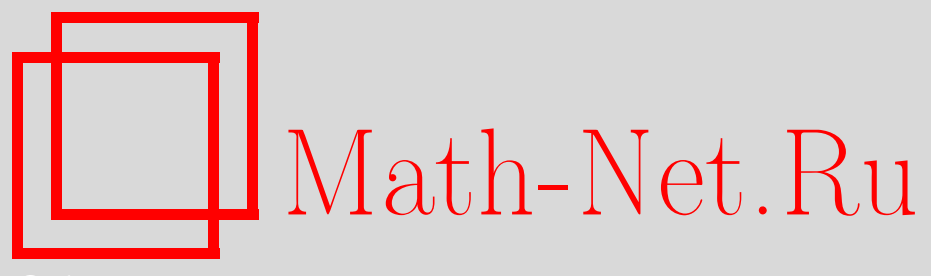

А. Г. Петрова, Об условиях монотонности свободной границы в двухфазной задаче Стефана, Матем. заметки, 2013, том 93, выпуск 5, 798-800

DOI: https://doi.org/10.4213/mzm9285

Использование Общероссийского математического портала Math-Net.Ru подразумевает, что вы прочитали и согласны с пользовательским соглашением http: //www . mathnet.ru/rus/agreement

Параметры загрузки:

IP : 34.227 .88 .159

26 апреля 2023 г., 09:45:01

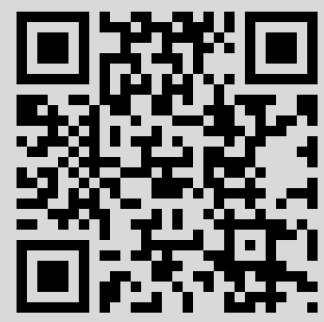




\section{Об условиях монотонности свободной границы в двухфазной задаче Стефана}

\section{А. Г. Петрова}

Результаты о монотонности свободной границы в однофазной задаче Стефана (см., например, [1]) давно и хорошо известны. Широко используемый в приложениях процесс направленной кристаллизации в двухфазном случае реализуется лишь в случае монотонности фронта. Принадлежащая автору заметка, в которой были сформулированы достаточные условия монотонности свободной границы в двухфазной задаче и приведена схема доказательства, была опубликована в малодоступном издании Института гидродинамики СО РАН "Динамика сплошных сред” в 1984 г. [2]. В 2011 г. В. С. Белоносовым были высказаны сомнения в возможности доказать заявленный в [2] результат без дополнитеных условий, обеспечивающих аналитичность свободной границы. В данной заметке приведено развернутое доказательство результата, не использующее аналитичности.

Рассмотрим одномерную двухфазную задачу Стефана нахождения функций $u_{i}(x, t)$, $i=s, l$, в областях

$$
\Omega_{s}(t)=\{x: 0<x<s(t)\}, \quad \Omega_{l}(t)=\{x: s(t)<x<1\}
$$

соответственно, а также функции $s(t)$, определяющей положение фазовой границы в момент времени $t$ по следующим условиям:

$$
\begin{gathered}
\alpha_{i} \frac{\partial u_{i}}{\partial t}=\frac{\partial^{2} u_{i}}{\partial x^{2}}, \quad x \in \Omega_{i}(t), \quad t \in(0, T) ; \\
u_{s}=u_{l}=0, \quad \gamma \dot{s}(t)=\frac{\partial u_{s}}{\partial x}-\frac{\partial u_{l}}{\partial x}, \quad x=s(t), \\
u_{s}(0, t)=f_{s}(t), \quad u_{l}(1, t)=f_{l}(t), \\
u_{i}(x, 0)=\phi_{i}(x), \quad x \in \bar{\Omega}_{i}(0), \quad i=s, l, \quad s(0)=s_{0} \in(0,1) .
\end{gathered}
$$

Нетрудно видеть, что классическая двухфазная задача Стефана может быть приведена к виду (1), (2), в котором коэффициенты при производных по пространственной переменной как в уравнении теплопроводности, так и в условии Стефана равны единице. Здесь $\alpha_{i}$, $\gamma$ - положительные константы, $f_{i}(t)$ - непрерывно дифференцируемые, а $\phi_{i}(x)$ - дважды непрерывно дифференцируемые функции такие, что

$$
\begin{gathered}
\phi_{s}<0, \quad x \in \Omega_{s}(0), \quad \phi_{l} \geqslant 0, \quad x \in \Omega_{l}(0), \\
\phi_{i}\left(s_{0}\right)=0, \quad f_{s}(t)<0, \quad f_{l}(t)>0, \quad t \in[0, T], \quad f_{s}(0)=\phi_{s}(0), \quad f_{l}(0)=\phi_{l}(1), \\
\alpha_{s} f_{s}^{\prime}(0)=\phi_{s}^{\prime \prime}(0), \quad \alpha_{l} f_{l}^{\prime}(0)=\phi_{s}^{\prime \prime}(1), \quad \gamma \dot{s}(0)=\phi_{s}^{\prime}\left(s_{0}\right)-\phi_{l}^{\prime}\left(s_{0}\right) . \\
\alpha_{i} \phi_{i}^{\prime}\left(s_{0}\right) \dot{s}(0)+\phi_{i}^{\prime \prime}\left(s_{0}\right)=0, \quad i=s, l .
\end{gathered}
$$

Вопросы существования классического решения одномерной двухфазной задачи Стефана давно исследованы [1], [3]; нас будет интересовать свойство классического решения монотонность фазового фронта.

Теорема 1. Пусть данные задачи (1)-(4) помимо условий (5) удовлетворяют следующим требованиям:

$$
\begin{aligned}
\dot{s}(0)>0, \quad \phi_{s}^{\prime \prime}(x) & <0, \quad x \in \bar{\Omega}_{s}(0), \quad \phi_{l}^{\prime \prime}(x) \leqslant 0, \quad x \in \bar{\Omega}_{l}(0), \\
f_{s}^{\prime}(t) & <0, \quad f_{l}^{\prime}(t) \leqslant 0, \quad t \in[0, T] .
\end{aligned}
$$

DOI: $10.4213 / \mathrm{mzm} 9285$ 
Тогда функиия $s(t)$ является строго возрастающей функиией на всем интервале сущесвования классического решения $(0, T)$ u $\dot{s}(t)>0, t \in(0, T)$.

ДоказАтельство. Прежде всего заметим, что $s(t)$ бесконечно дифференцируема на интервале $(0, T)$, а функции $u_{i}(x, t)$ являются бесконечно дифференцируемыми внутри областей $Q_{i}(T)=\left\{(x, t): x \in \Omega_{i}(t), t \in(0, T)\right\}[3]$ и в силу принципа максимума, примененного к функциям $u_{i}$, имеем

$$
\left.\frac{\partial u_{i}}{\partial x}\right|_{x=s(t)} \geqslant 0, \quad t \in(0, T)
$$

Предположим, что существуем $t^{*} \in(0, T)$ такое, что $\dot{s}(t)>0$ при $t<t^{*}$ и $\dot{s}\left(t^{*}\right)=0$. Вследствие равенств

$$
\frac{\partial u_{i}}{\partial x}(s(t), t) \dot{s}(t)+\frac{\partial u_{i}}{\partial t}(s(t), t)=0
$$

и оценок (6) имеем

$$
\frac{\partial u_{i}}{\partial t}(s(t), t) \leqslant 0, \quad t \in\left[0, t^{*}\right] .
$$

Отметим, что в силу леммы о знаке производной [1] имеет место строгое неравенство

$$
\left.\frac{\partial u_{s}}{\partial x}\right|_{x=s(t)}>0, \quad t \in[0, T) .
$$

Из $(7),(8)$ и предположения о точке $t^{*}$ следует, что

$$
\frac{\partial u_{s}}{\partial t}(s(t), t)<0, \quad t \in\left[0, t^{*}\right), \quad \frac{\partial u_{s}}{\partial t}\left(s\left(t^{*}\right), t^{*}\right)=0 .
$$

Отсюда, принимая во внимание условия на граничные и начальные функции задачи, заключаем, что $\partial u_{s} / \partial t$ достигает в $\bar{Q}_{s}\left(t^{*}\right)=\left\{x: 0 \leqslant x \leqslant s(t), 0 \leqslant t \leqslant t^{*}\right\}$ своего строгого максимума, равного нулю, в точке $\left(s\left(t^{*}\right), t^{*}\right)$.

Покажем, что

$$
\frac{\partial^{2} u_{s}}{\partial t \partial x}\left(s\left(t^{*}\right), t^{*}\right)>0 .
$$

Выберем $\bar{x} \in\left(0, s\left(t^{*}\right)\right)$ так, чтобы замкнутое множество

$$
\bar{B}=\left\{(x, t):(x-\bar{x})^{2}+\left(t-t^{*}\right)^{2} \leqslant R^{2}, t^{*}-\delta \leqslant t \leqslant t^{*}, \frac{\bar{x}+s\left(t^{*}\right)}{2} \leqslant x \leqslant s(t)\right\},
$$

где $R=s\left(t^{*}\right)-\bar{x}, 0<\delta<R$, содержалось в $\bar{Q}_{s}\left(t^{*}\right)$. Существование такого множества $\bar{B}$ обеспечивается бесконечной дифференцируемостью функции $s(t)$ при $t \in(0, T)[3]$.

Введем функцию

$$
v=\frac{\partial u_{s}}{\partial t}+\epsilon h
$$

где

$$
h(x, t)=\exp \left(-\alpha\left((x-\bar{x})^{2}+\left(t-t^{*}\right)^{2}\right)\right)-\exp \left(-\alpha R^{2}\right) .
$$

Имеем $h=0$ на криволинейной границе, представляющей собой дугу окружности радиуса $R$, и $h_{x x}-h_{t}>0$ внутри области $B$, если $\alpha$ достаточно большое. Для любого достаточно малого $\epsilon>0$ функция $v$ будет меньше нуля на прямолинейных участках $x=\left(\bar{x}+s\left(t^{*}\right)\right) / 2$ и $t=t^{*}-\delta$ границы области $\bar{B}$. Таким образом, функция $v$ достигает своего максимума в области $\bar{Q}_{s}\left(t^{*}\right)$ в точке границы с координатами $\left(s\left(t^{*}\right), t^{*}\right)$. Итак, $v_{x}\left(s\left(t^{*}\right), t^{*}\right) \geqslant 0$. Отсюда получаем

$$
\frac{\partial^{2} u_{s}}{\partial t \partial x}\left(s\left(t^{*}\right), t^{*}\right)=v_{x}\left(s\left(t^{*}\right), t^{*}\right)-\epsilon\left(-2 \alpha\left(s\left(t^{*}\right)-\bar{x}\right) \exp \left(-\alpha\left(s\left(t^{*}\right)-\bar{x}\right)^{2}\right)>0 .\right.
$$


В силу неположительности функции $\partial u_{l} / \partial t$ в $\bar{Q}_{l}\left(t^{*}\right)$, вытекающей из принципа максимума, а также равенства ее нулю при $t=t^{*}$ имеем оценку

$$
\frac{\partial^{2} u_{l}}{\partial t \partial x}\left(s\left(t^{*}\right), t^{*}\right) \leqslant 0 .
$$

Рассмотрим $\ddot{s}\left(t^{*}\right)$. Из условия $\dot{s}\left(t^{*}\right)=0$ и оценок $(9),(10)$ следует, что

$$
\gamma \ddot{s}\left(t^{*}\right)=\frac{\partial^{2} u_{s}}{\partial t \partial x}\left(s\left(t^{*}\right), t^{*}\right)-\frac{\partial^{2} u_{l}}{\partial t \partial x}\left(s\left(t^{*}\right), t^{*}\right)>0,
$$

что противоречит предположению о том, что $\dot{s}(t)>0$ при $t \in\left[0, t^{*}\right)$ и $\dot{s}\left(t^{*}\right)=0$. Полученное противоречие доказывает теорему.

Автор благодарит профессоров Г. И. Бижанову, А. М. Мейрманова и Е. В. Радкевича за полезное обсуждение этого доказательства.

\section{СПИСОК ЦИТИРОВАННОЙ ЛИТЕРАТУРЫ}

[1] А. Фридман, Уравнения с частными производными параболического типа, М., Наука, 1968. [2] А. Г. Петрова, Динамика сплошной средъ, 67 (1984), 97-99. [3] А. М. Мейрманов, Задача Стефана, Новосибирск, Наука, 1986.

\section{А. Г. Петрова}

Поступило

Алтайский государственный университет,

02.12 .2011

г. Барнаул

E-mail: annapetrova07@mail.ru 